EFFECT OF DECARBONIZATION OF METAL ON THE LOAD-

CARRYING CAPACITY OF CYLINDRICAL PRESSURE VESSELS

V. P. Shirshov

UDC $\in 2-98: 620.165$

The recommendations of Kotlonadzor [1] on evaluating the strength of parts made of cast steel do not explicity deal with the effect of decarbonization. Nevertheless, the effect can be differentiated for parts of different thicknesses, since the depth of decarbonization is, generally speaking, controlled in relation to the thickness of the walls of the casting.

In [2] we determined the breaking pressure for the cylindrical wall of a steel vessel which contains some decarbonized metal. This problem is important for cast structures in which, for technical reasons, the percentage of decarbonized metal may reach about $30 \%$.

Without additional explanation, we will use the notation in [2]. We note only that the quantities with an asterisk pertain to decarbonized metal.

The thickness of the gage (momentless) wall and the thickness of the base and decarbonized layers of metal will be assumed constant. We will further suppose that the base and decarbonized metal are homogeneous and isotropic, and that the elastic constants are the same. The stress-strain curves will be represented in the form [1]

$$
\sigma_{i}=\sigma_{s}\left(\frac{\varepsilon_{i p c}}{\delta_{s}}\right)^{\alpha} ; \quad \sigma_{i}^{*}=\sigma_{s}^{*}\left(\frac{\sigma_{i p c}^{*}}{\delta_{s}}\right)^{\alpha} \text {, }
$$

where

$$
\varepsilon_{i \mathrm{pc}}=\varepsilon_{i}-\frac{\sigma_{i}}{E_{i}} ; \quad \varepsilon_{i \mathrm{pc}}^{*}=\varepsilon_{i}^{*}-\frac{\sigma_{i}^{*}}{E_{i}} .
$$

The stress-strain state of the shell will be subject to the conditions

$$
\begin{gathered}
\frac{\sigma_{1}}{\sigma_{2}}=\frac{\sigma_{1}^{*}}{\sigma_{2}^{*}}=\frac{1}{2} ; \\
\varepsilon_{2}^{*}=\varepsilon_{2} ; \quad \varepsilon_{3}^{*}=\varepsilon_{3} ; \quad \varepsilon_{i \mathrm{pc}}^{*}=\varepsilon_{i \mathrm{pc}}
\end{gathered}
$$

With allowance for (3-4), we write the stress-strain relations in the form

$$
\varepsilon_{2}=\frac{\sqrt{3}}{2} \varepsilon_{i \mathrm{pc}}+\frac{2-\mu}{2} \frac{\sigma_{2}}{E} ; \quad \varepsilon_{3}=-\frac{\sqrt{3}}{2} \varepsilon_{i \mathrm{pc}}-\frac{3 \mu}{2} \frac{\sigma_{2}}{E},
$$

while from the equilibrium equation

and the relations

$$
\sigma_{2} h+\sigma_{2}^{*} h^{*}=p \cdot r
$$

$$
h=h_{0} e^{\varepsilon_{s}} ; \quad h^{*}=h_{0} e^{e_{3}^{*}} ; \quad r=r_{0} e^{\varepsilon_{2}},
$$

where $h_{0}, h_{0} * ; h, h^{*}$ are the thicknesses of the base and decarbonized (summed from both sides) metal in the initial (with the subscript " 0 ") and loaded states, we obtain the expression

$$
p=\frac{h_{0} \sigma_{2}+h_{0}^{*} \cdot \sigma_{2}^{*}}{r} \cdot e^{\mathrm{e}_{3}-\mathrm{e}_{2}} .
$$

First we determine the limiting pressure $P_{m t}$ due to strain localization. Here, in (5) we discard the terms with elastic constants and we transform Eq. (6) to the following form, with allowance for (3)

$$
F=\frac{2}{\sqrt{3}} \frac{h_{0} \sigma_{i}+h_{0}^{*} \cdot \sigma_{i}^{*}}{r_{0}} e^{-\sqrt{3} \cdot \varepsilon_{i} \mathrm{pc}}
$$

Tula. Translated from Problemy Prochnosti, No. 8, pp. 72-74, July, 1985. Original article submitted July $12,1983$. 
We obtain the value of $p_{m t}$ from the condition $d p / d \varepsilon_{i p c}=0$, which we write as follows with allowance for (1) and (4)

$$
\left(\varepsilon_{i p c}-\frac{\alpha}{\sqrt{3}}\right) \sigma_{i} h_{0}=\left(\frac{\alpha^{*}}{\sqrt{3}}-\varepsilon_{i p c}\right) \sigma_{i} \cdot h_{0}^{\dot{0}}
$$

To obtain an approximate solution of Eq. (8) we use a linear approximation of Eq. (1) In the range $\frac{\alpha}{\sqrt{3}} \leqslant \varepsilon_{i p c} \leqslant \frac{\alpha^{*}}{\sqrt{3}}$, which is bounded by the limiting values [2] of the quantity $\varepsilon_{i p c}$ for uniform shells made up of base and decarbonized metals:

$$
\sigma_{i}=\sigma_{i \mathrm{mt}}+B\left(\varepsilon_{i \mathrm{pc}}-\frac{\alpha}{\sqrt{3}}\right) ; \quad \sigma_{i}^{*}=\sigma_{i \mathrm{mt}}^{*}+B^{*}\left(\varepsilon_{i \mathrm{pc}}-\frac{\alpha^{*}}{\sqrt{3}}\right),
$$

where

$$
\begin{aligned}
& B=\sqrt{3} \cdot \sigma_{s} \frac{\left(\frac{\alpha^{*}}{\delta_{s} \sqrt{3}}\right)^{\alpha}-\left(\frac{\alpha}{\delta_{s} \cdot \sqrt{3}}\right)^{\alpha}}{\alpha^{*}-\alpha} ; \\
& B^{*}=\sqrt{3} \sigma_{s}^{*} \frac{\left(\frac{\alpha^{*}}{\delta_{s} \cdot \sqrt{3}}\right)^{\alpha *}-\left(\frac{\alpha}{\delta_{s} \sqrt{3}}\right)^{\alpha *}}{\alpha^{*}-\alpha} ; \\
& \sigma_{i \mathrm{mr}} \cdot \sigma_{s}\left(\frac{\alpha}{\delta_{s} \sqrt{3}}\right)^{\alpha} ; \quad \sigma_{i \mathrm{mt}}^{*}=\sigma_{s}^{*}\left(\frac{\alpha^{*}}{\sigma_{s} \sqrt{3}}\right)^{\alpha}
\end{aligned}
$$

The quantities $\sigma_{i \mathrm{mt}}$ and $\sigma_{i \mathrm{mt}}^{*}$ represent the values [2] of stress intensity corresponding to strain localization in uniform shells made of base and decarbonized metal, respectively.

Having made the substitution of variables

$$
\varepsilon_{i p c}=\frac{e}{\sqrt{3}}(1+\beta): \quad \beta=\frac{\alpha^{*}-\alpha}{\alpha} i
$$

we write Eq. (8) in the following form with allowance for (9-11)

$$
a \gamma^{2}+b y+c=0
$$

where

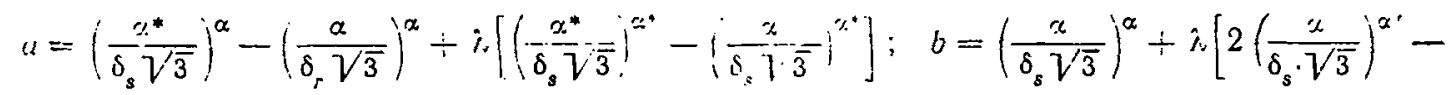

$$
\begin{aligned}
& -\left(\frac{\alpha^{*}}{\delta_{s} \sqrt{3}}\right)^{\alpha *} \mid ; \quad c=-\lambda\left(\frac{\alpha}{\delta_{s} \sqrt{3}}\right)^{\alpha} ; \\
& \lambda=\frac{h_{0} \cdot \sigma_{s}^{*}}{h_{0} \cdot \sigma_{s}} \text {. }
\end{aligned}
$$

Having inserted (12) into (7) with allowance for (1), we obtain the formula

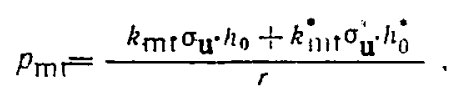

where

$$
\begin{aligned}
& \left.k_{\mathrm{mT}}=\frac{2}{\sqrt{3}} \frac{\pi_{s}}{\sigma_{\mathrm{u}}} \mid \frac{\alpha}{\delta_{s} \sqrt{3}} \cdot \frac{1+\beta}{e^{1-\beta}}\right)^{x} ; \\
& k_{\mathrm{ml}}^{*}=\frac{2}{1-\bar{s}} \frac{\sigma_{s}^{*}}{\sigma_{u}^{*}}\left(\frac{\%}{\sigma_{s}} \cdot \frac{1-\hat{\beta}}{1 \overline{3}}\right)^{\alpha^{*}} \cdot e^{-\alpha_{c}(1-\beta)} .
\end{aligned}
$$

We determine the breaking pressure $\mathrm{p}_{\mathrm{b}}<\mathrm{P}_{\mathrm{mt}}$ from the rupture of the wall through the base metal (the decarbonized metal is more ductile). This rupture occurs at a stress [2] equal to

$$
\sigma_{2}=\sigma_{\mathrm{u}} \cdot e^{\delta}
$$
formula

Having inserted (15) and (5) into (7), with allowance for (3) and (1) we obtain the

$$
p_{\mathrm{b}}=\frac{k_{\mathrm{h}} \cdot \sigma_{\mathrm{u}} \cdot h_{0}+k_{\mathrm{h}} \cdot h_{0} \cdot \sigma_{\mathrm{u}}^{*}}{r_{0}}
$$




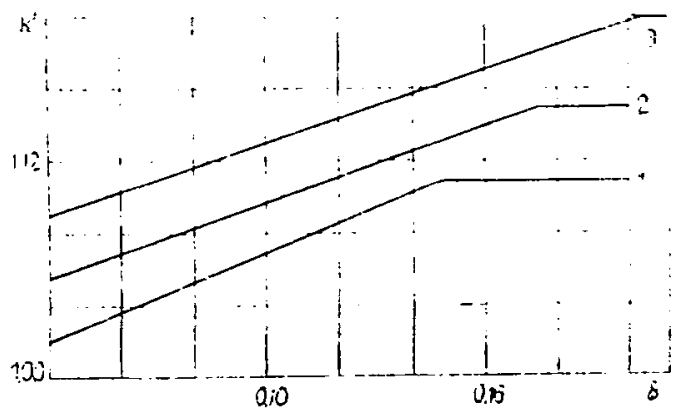

Fig. 1. Dependence of the coefficient $k^{\prime}$ on elongation and the depth of the decarbonized metal: $1-\frac{l_{1}}{i_{i}}=\because, 2-\frac{h_{0}^{*}}{h_{n}}=0.15 ; 3-\frac{i_{0}^{*}}{h_{\mathrm{a}}}-n .3$.

where

$$
\begin{gathered}
k_{\mathrm{h}}=\exp \left[\delta-l \cdot \overline{3} \delta_{\mathrm{s}} \cdot \vartheta^{1 / \alpha}-(1+\mu) \frac{\sigma_{\mathrm{u}}}{E} e^{\delta}\right] ; \\
k_{\mathrm{h}}=\frac{2}{\sqrt{3}} \cdot \frac{\sigma_{s}^{*}}{\sigma_{\mathrm{u}}} \vartheta^{x^{*} / \alpha} \cdot \exp \left[-1 \cdot \overline{3} \delta_{\delta} \vartheta^{1 / \alpha}--(1+\mu) \frac{\sigma_{\mathrm{u}}}{E} e^{\delta}\right] ; \quad \vartheta=\frac{\sqrt{3}}{2} \cdot \frac{\sigma_{\mathrm{u}} \cdot e^{\delta}}{\sigma_{\mathrm{s}}} .
\end{gathered}
$$

Equations (16) and (17) should be used in the case $e_{i_{b}}<e_{i_{t}}$, where $\varepsilon_{i_{b}}$ and $\varepsilon_{i_{m t}}$ are the values of $\varepsilon_{i}$ at wall rupture and strain localization, respectively. When $\varepsilon_{i b}>\varepsilon_{i m t}$, it is necessary to use Eqs. (13) and (14). We find from (12), (2), and (1) that

$$
\varepsilon_{i \mathrm{mr}}=\frac{\alpha}{\sqrt{3}}(1+\beta) \div \frac{2(1+\mu)}{V^{\prime}}\left(\frac{\alpha}{\sqrt{3}} \cdot \frac{1+\beta}{\delta_{s}}\right)^{6} \frac{\dot{\alpha}}{\dot{E}}
$$

The following formula is valid for $\varepsilon_{i b}$ [2]

$$
\varepsilon_{i \mathrm{~b}}=\delta_{3}\left(\frac{\sqrt{3}}{\underline{\underline{a}}} e^{\delta} \frac{\sigma_{\mathrm{u}}}{\sigma_{\mathrm{s}}}\right)^{1 / \alpha}+\frac{1+\mu}{\sqrt{3}} e^{s} \frac{\sigma_{\mathrm{u}}}{E} .
$$

As an example, let us examine the structural strength of a vessel made of steel (with the following mechanical characteristics: $\sigma_{\mathrm{u}}=1500 \mathrm{MPa} ; \sigma_{\mathrm{s}}=\sigma_{0.2}=1250 \mathrm{MPa} ; \sigma_{\mathrm{u}}{ }^{*}=500$ $\mathrm{MPa} ; \sigma_{\mathrm{S}} *=\sigma_{0.2} *=250 \mathrm{MPa}$ ) in relation to the elongation $\delta$ of the base metal and the relative depth of decarbonization $h_{0}^{*} / h_{0}$.

To this end, we represent the breaking pressure in the form

$$
P_{\mathrm{frac}}=k^{\prime} \frac{\sigma_{\mathbf{u}} \cdot h_{0}}{r_{0}},
$$

where

$$
k^{\prime}=k+k^{*} \frac{\sigma_{\mathbf{u}^{\prime}}^{*} \cdot h_{0}^{*}}{\sigma_{\mathbf{u}^{*} \cdot !_{0}}},
$$

where $k$ and $k *$ are determined from either (14) or (17), in accordance with the above conditions.

We will consider the ductility of the decarbonized metal to be sufficient for strain localization both in the vessel wall and in the tension of a fully decarbonized straight specimen. On the basis of this, we obtain $\alpha^{*}=0.194$ from Eq. (13) in [2]. We will also borrow the relation $\alpha(\delta)$ for the base metal from [2].

The values of $\mathrm{k}^{\prime}(\delta)$ calculated with allowance for the foregoing are shown in Fig. 1 for three values of $h_{0}^{*} / h_{0}: 0 ; 0.15 ; 0.3$. They were constructed in the same manner as the relation $k(\delta)$ in [2] for a vessel made of a uniform material, with which the curve $k^{*}(\delta)$ coincides at $h_{0}^{*} / h_{\alpha}=0$. It is evident from the flgure that allowing for the decarbonized metal in the calculation produces a safety factor of up to about $10 \%$.

It should be noted that the decarbonized metal has no appreciable effect on the structural-strength coefficients $k_{b}$ and $k_{m t}$ of the base layer. These coefficients are nearly independent of $h_{0}^{*} / h_{0}$ in the investigated range of $h_{0}^{*} / h_{0}=0-0.3$. 
The method used here reliably reflects the effect of decarbonization on the strength of the cylindrical walls of pressure vessels, as was confirmed in tests: experimental cylinders free of any defects that might affect their strength had breaking pressures which were consistent with the values calculated by the above method. The cylinders fractured through the cylindrical walls.

\section{LITERATLRE CITED}

1. Handbook of Kotlonadzor Projects [in Russian], Énergiya, Moscow (1974).

2. V. P. Shirshov, "Prediction of fracture of thin-walled pressure vessels," Probl. Prochn., No. 6, 60-63 (1982).

EFFECT OF THE PEAK TEMPERATURES AND OF THE COOLING

RATE ON THE DAMPING PROPERTIES OF MANGANESE-

\section{COPPER BASE ALLOYS}

B. S. Chaikovskii, G. Ya, Yaroslavskii,

UDC $539.67 ; 620.178$

L. A. Bocharova, O. T. Bashta,

and V. V. Matveev

It was shown in $[1,2]$ that alloys of the system $\mathrm{Mn}-\mathrm{Cu}-\mathrm{Zn}$ are distinguished by a favorable combination of high damping ability, strength, and ductility. It is, however, very lmportant that actual products made of these alloys have a high level of energy losses.

When structures are produced, the alloy may be subjected to various thermal effects (e.g., in consequence of the technological operation of welding) which may change its initial damping properties.

To determine the effect of various peak temperatures and cooling rates on the damping ability of copper-manganese alloys at normal temperature, we carried out the respective tests on an installation KD-1 [3].

We investigated alloys differing in chemical composition as well as by the regimes of heat treatment in the initial state (before heat cycling). Heat cycling consisted in heating blanks of a copper-manganese alloy to $200-850^{\circ} \mathrm{C}$ with subsequent cooling at rates of 5 to $150-200^{\circ} \mathrm{C} / \mathrm{sec}$. After the heat cycling, specimens for investigating the damping properties were made from the blanks.

Heat cycling was applied to alloys in different initial states (cast, hardened, hardened + tempered). The chemical composition of the alloys concerned is presented in Table 1.

The investigations showed (Fig. 1) that heating from 200 to $400^{\circ} \mathrm{C}$ and subsequent cooling at rates from 5 to $150-200^{\circ} \mathrm{C} / \mathrm{sec}$ has practically no effect on the damping ability of the alloy in the cast state. Further heating to 600 or $850^{\circ} \mathrm{C}$ with the same cooling rates causes a noticeable lowering of the level of the dissipative properties of the alloy (by a factor of $1.5-2$ ).

The cooling rate has practically no effect on the damping properties of the investigated alloys in the cast state after they have been heated to between 200 and $850^{\circ} \mathrm{C}$. However, the peak temperature to which the alloy is heated causes a substantial change of its dissipative properties independently of the cooling rate. The greatest change in the damping ability of the alloys in the cast state is found when they are heated to $600^{\circ} \mathrm{C}$ or more.

Alloys of the system $\mathrm{Mn}-\mathrm{Cu}-\mathrm{Zn}$ in the cast state are characterized by general inhomogeneity and liquation in regard to the chemical composition, and the cooling conditions have the effect that the structure of the alloys, together with $\gamma(\mathrm{fcc})$-phase, also contains the tetragonal $\gamma_{t}$-phase whose presence is decisive for the high damping of these alloys. The $\gamma_{t}$-phase is distributed nonuniformly throughout the bulk, and in consequence damping is not

Institute of Strength Problems, Academy of Sciences of the Ukrainian SSR. Kiev Polytechnic Institute, Kiev, Leningrad. Translated from Problemy Prochnosti, No. 8, pp. 74-77, August, 1985. Original article submitted June 14, 1984. 